\title{
Brustkrebs: Update zur kardialen Sicherheit unter Trastuzumab
}

\begin{abstract}
Der monoklonale Antikörper Trastuzumab hat sich als tragende Säule in der adjuvanten Therapie des HER2-positiven Mammakarzinoms etabliert. Dem möglicherweise erhöhten Risiko für kardiotoxische Nebenwirkungen kommt daher große Bedeutung zu.
\end{abstract}

\begin{abstract}
n einer gemeinsamen Analyse der adjuvanten Studien NCCTG N9831 (Alliance) und NSABP B-31 (NRG) verbesserte eine 1-jährige Trastuzumab-Addition zu Doxorubicin/Cyclophosphamid (AC) und nachfolgend Paclitaxel krankheitsfreies und Gesamtüberleben. Die Forscher legten bereits eine Analyse der N9831-Studie zur kardialen Sicherheit nach median 3,75 Jahren vor. Im Rahmen dieser Studie erhielten Patientinnen mit operablem, HER2-positivem Mammakarzinom vier Zyklen AC, gefolgt von nur Paclitaxel (Arm A), Paclitaxel und sequenziell Trastuzumab (Arm B) oder gleichzeitig Paclitaxel plus Trastuzumab (Arm C). Die kumulative Rate für die 3-Jahres-Inzidenz kardialer Ereignisse
\end{abstract}

(CE) betrug in den Armen jeweils 0,3\%, $2,8 \%$ und $3,3 \%$. Angesichts der überzeugenden Wirksamkeit von Trastuzumab aktualisierten die Forscher die kardiale Sicherheitsanalyse von N9831 nach median 9,2 Jahren und verwendeten einen kardialen Risikoscore.

Zur Analyse der kumulativen CE-Inzidenz und der linksventrikulären Ejektionsfraktion (LVEF) eigneten sich 1.944 Patientinnen mit einer Post-AC-Therapie. 651 willigten einer Reevaluierung der LVEF nach median 6 Jahren ein. Es ergab sich eine absolute mediane Änderung der LVEF gegenüber dem Ausgangswert um $-3,0 \%$ in Arm A, $-2,5 \%$ in Arm B und $-3,0 \%$ in Arm C. Die kumulative CE-Inzidenz nach 6 Jahren betrug in Arm A
$0,6 \%$, in den Armen B und C 2,8\% bzw. $3,4 \%$. Nach median 9,2 Jahren ergaben sich nur zwei zusätzliche Fälle von Herzinsuffizienz (CHF) gegenüber dem 3,75-jährigen Follow-up. Die LVEF erholte sich bei den meisten CHF-Patientinnen wieder. Ein Alter $>60$ Jahre, eine LVEF $<65 \%$ und die Einnahme von Antihypertensiva korrelierten mit einem erhöhten Risiko für CE in den Armen B und C.

Fazit: Die kumulative Inzidenz von CE erhöht sich geringfügig durch die Erweiterung einer adjuvanten Chemotherapie um Trastuzumab. Späte kardiale Komplikationen treten selten auf. Der Nutzen von Trastuzumab im Kontext einer Anthrazyklin- und Taxan-basierten Chemotherapie überwiegt daher weiterhin das Risiko. Wolfgang Zimmermann

Advani PP et al. Long-Term Cardiac Safety Analysis of NCCTG N9831 (Alliance) Adjuvant Trastuzumab Trial. J Clin Oncol. 2016;34(6):581-7.

\section{FDG-PET/CT verbessert Stratifizierung für Strahlentherapie beim Mammakarzinom}

\author{
Die ${ }^{18} \mathrm{~F}$-FDG-PET/CT könnte das Staging vor Beginn einer Therapie von \\ Patientinnen mit lokal fortgeschrittenem Brustkrebs verbessern. In einer \\ Studie wurde diese Methode mit konventioneller Bildgebung als Basis für \\ die Entscheidung zu einer Strahlentherapie verglichen.
}

$P_{\text {tient }}^{\text {rosp }}$ rospektiv schlossen die Forscher Patientinnen mit lokal fortgeschrittenem Mammakarzinom in ihre Studie ein, die noch einen guten bis mäßigen Allgemeinzustand (ECOG[Eastern Cooperative Oncology Group]-Performancestatus $<2$ ) und keine Kontraindikationen für eine neoadjuvante Chemotherapie, chirurgische Therapie oder adjuvante Strahlentherapie aufwiesen. Bei allen $\mathrm{Pa}-$ tientinnen war vor Therapiebeginn eine umfassende konventionelle Bildgebung (CI) mit beidseitiger Mammografie und Ultraschalluntersuchung sowie einer Computertomografie von Brust, Abdomen und Becken erfolgt. In die Auswertung gingen 154 Patientinnen (median
26-70 Jahre) ein, bei denen klinisch und radiologisch in der CI kein Anzeichen von Fernmetastasen gefunden worden war. Mit der zusätzlich durchgeführten ${ }^{18} \mathrm{~F}$-Fluorodeoxyglukose-Positronenemissionstomografie/Computertomografie (F-FDG-PET/CT) des gesamten Körpers wurden bei 32 Patientinnen $(20,8 \%)$ Fernmetastasen und/oder eine lokoregionale Erkrankung identifiziert, die vorher anhand der CI nicht erkannt worden waren. Fernmetastasen wurden bei 17 Patientinnen (11,0\%) gefunden: Knochenmetastasen bei 6 Patientinnen, intrathorakale Metastasen (in Lunge und Mediastinum) bei 5, entfernt liegende Lymphknotenmetastasen bzw. Leberme- tastasen bei jeweils 2 Patientinnen und je eine Patientin wies eine Kombination von Lungen- und Knochenmetastasen bzw. mediastinalen und distalen Lymphknotenmetastasen auf. Bei 15 (10,8\%) der übrigen 139 Patientinnen wurde durch die F-FDG-PET/CT zusätzlich eine Lymphknotenmetastasierung entdeckt, die außerhalb des konventionellen Bestrahlungsfeldes lag.

Fazit: Das F-FDG-PET/CT kann bei Patientinnen mit lokal fortgeschrittenem Brustkrebs wesentliche Informationen zu Diagnose und Staging beisteuern, die über die CI hinausgehen und relevant sind für die Entscheidung zur Bestrahlung. Die Forscher empfehlen daher, bei allen entsprechenden Patientinnen vor Therapie eine PET/CT durchzuführen.

Friederike Klein

$\mathrm{Ng}$ SP et al. Impact of Pretreatment Combined (18)F-Fluorodeoxyglucose Positron Emission Tomography/Computed Tomography Staging on Radiation Therapy Treatment Decisions in Locally Advanced Breast Cancer. Int J Radiat Oncol Biol Phys. 2015;93(1):111-7. 